\title{
Respuesta orgánica en una simulación de combate
}

\author{
Clemente Suárez VJ. ${ }^{1}$, Robles Pérez JJ. ${ }^{2}$
}

Sanid. mil. 2012; 68 (2): 97-100; ISSN: 1887-8571

\begin{abstract}
RESUMEN
Antecedentes y objetivos: El análisis de la respuesta orgánica durante el combate es un campo de estudio poco tratado en la bibliografía específica, por ello se planteo como objetivo de este trabajo el analizar el efecto de una simulación de combate en el organismo de los combatientes. Material y Métodos: Se analizó el umbral de frecuencia crítica de fusión (UFCF) como medio de detectar fatiga del sistema nervioso central (SNC), fuerza explosiva de piernas, fuerza isométrica, lactato sanguíneo y percepción subjetiva de esfuerzo en 20 combatientes antes y después de realizar una simulación de combate. Resultados: Después de la simulación los sujetos mostraron un aumento significativo $(\mathrm{p}<0,05)$ en los valores de lactato $(2,57 \pm 0,62 \mathrm{mmol} / \mathrm{l} \mathrm{vs} .4,92 \pm 2,18 \mathrm{mmol} / \mathrm{l})$ y de fuerza isométrica y una disminución en la fuerza explosiva de piernas y los valores de UFCF. Conclusiones: La simulación de combate fue altamente estresante, mostrando síntomas de fatiga del SNC, se realizan en condiciones anaeróbicas, a pesar de la baja velocidad de ejecución y la percepción subjetiva de esfuerzo no se corresponde con la respuesta orgánica obtenida. Estos datos sirven para adecuar los actuales sistemas de entrenamiento para las actuales misiones en zonas de combate.
\end{abstract}

PALABRAS CLAVE: Lactato, Combate, Combatiente, Fuerza, Frecuencia crítica de fusión.

\section{Organic response to a combat simulation}

SUMMARY

Background and Objectives: Analysis of organic response in combat is a bit field of study specifically studied in specific literature, for this reason the objective of this work is to analyze the effect of a combat simulation in the organism of soldiers. Material and Methods: We analyzed the critical flicker fusion frequency (CFFF) as a means of detecting fatigue of central nervous system (CNS), leg explosive strength, isometric strength, blood lactate and rating of perceived exertion in 20 soldiers before and after performing a combat simulation. Results: After combat simulation, soldiers showed a significant increase $(\mathrm{p}<0,05)$ in lactate levels $(2,57 \pm 0,62$ $\mathrm{mmol} / 1$ vs. 4,92 $\pm 2,18 \mathrm{mmol} / \mathrm{l}$ ) and isometric strength and a decrease in legs explosive strength. CFFF values decreased, showing signs of fatigue of CNS. Conclusions: Combat simulation was highly stressful, showing signs of fatigue of the CNS, are carried out in anaerobic conditions, despite the low speed of execution, and the subjective perception of effort does not correspond to the organic response obtained. These data could use to adapting current training systems for current missions in combat zones.

KEYWORDS: Lactate, Combat, Soldiers, Strength, Flicker fusion frequency.

\section{INTRODUCCIÓN}

El análisis de situaciones de combate y la respuesta orgánica de los combatientes ha sido poco estudiada en la bibliografía especializada. Los estudios actuales se han centrado en analizar los procesos de toma de decisiones de los $\operatorname{mandos}^{1}$, la asimetría estratégica de los actuales campos de batalla $^{2}$, los sistemas de coordinación entre diferentes unidades dentro del campo de batalla ${ }^{3}$ o la coordinación entre las funciones de combate y los sofisticados sistemas de $\operatorname{armas}^{4}$, entre otros.

El estudio de la respuesta orgánica especifica de los combatientes a diferentes estímulos de combate, ya sean simulados o

${ }^{1}$ Universidad Castilla la Mancha. Departamento de Actividad Física y Ciencias del Deporte. Facultad CC Deporte. Toledo. España.

${ }^{2}$ Cap. Infantería. Escuela Central de Educación Física del Ejército de Tierra. Departamento de Combate Cuerpo a Cuerpo y Defensa Personal, Área de Deportes Militares. Toledo. España.

Dirección para correspondencia: Vicente Javier Clemente Suárez. Gabinete de Natacão. Facultade de Desporto. Universidade do Porto. Rua Dr. Plácido Costa 91. 4200-450. Porto. Portugal. Tfno.: 0034 649340680. e-mail: vicente.clemente@uclm.es

Recibido: 21 de noviembre de 2011

Aceptado: 7 de marzo de 2012 reales, se ha visto muy limitado y únicamente se han centrado en la investigación de diferentes parámetros orgánicos antes y después de la realización de distintas misiones. Dentro de esta línea de investigación Lester et $\mathrm{al}^{5}$ analizaron la composición corporal y el estado físico de 73 combatientes de infantería después de 13 meses de misión en Iraq observando como después de esta misión, tanto la fuerza de miembros superiores e inferiores aumentó ( $7 \%$ y $8 \%$ respectivamente), al igual que la potencia muscular (9\%), además el rendimiento aeróbico disminuyó un 13\% y la masa grasa aumentó un $9 \%$. También se ha comprobado como el porcentaje de grasa es un valioso indicador de rendimiento al analizar una prueba incremental de carrera con el equipo de combate $^{6}$. Rintamäki et al $^{7}$ observó como después de 12 días de maniobras militares realizadas en periodo invernal, no se produce fatiga acumulada en los soldados. Además no tiene efectos negativos en la fuerza máxima y el consumo de oxígeno máximo y produce una disminución de la frecuencia cardiaca de los combatientes, debida al entrenamiento realizado en estas maniobras. Otro de los campos de estudio más utilizado ha sido el análisis de diferentes test y su correlación con el rendimiento en diferentes situaciones de combate. Dentro de esta línea encontramos el estudio de Simpson et $\mathrm{al}^{8}$ que comprobaron la correlación entre 
el rendimiento en un test de 2 millas y el rendimiento en una marcha de $29 \mathrm{~km}$ por la montaña en dos unidades de élite del Ejército Británico. En este sentido también Harman et $\mathrm{al}^{9}$ mostraron como un test de $3,2 \mathrm{~km}$ y dos test de salto (horizontal y vertical) eran los mejores predictores de rendimiento en una batalla simulada.

Desligado de estos dos grupos de líneas de investigación y más acorde con el estudio que se plantea en este trabajo de investigación encontramos el trabajo de Jiméne ${ }^{10}$ que analizó la maniobra de avance de una unidad de infantería ligera desde la base de partida hasta las posiciones enemigas. Para ello simuló esta maniobra en un tapiz rodante donde analizó variables cardiorespiratorias. Los resultados mostraron un bajo nivel físico de los soldados y la necesidad de aumentar sus niveles de resistencia para una mejor ejecución de esta maniobra.

Después de esta revisión bibliográfica se puede observar como el estudio de la respuesta orgánica durante el combate es un área de conocimiento poco tratada en la bibliografía, a pesar de su capital importancia a la hora de poder realizar un correcto adiestramiento y preparación para dicho combate. Por todo ello planteamos como objetivo de este trabajo de investigación el estudio de la respuesta cortical, metabólica, de fuerza muscular y la percepción subjetiva de esfuerzo en una simulación de combate realizada en una pista de obstáculos de entrenamiento militar.

\section{MATERIAL Y MÉTODOS}

\section{Sujetos}

Se analizaron 20 miembros de las Fuerzas Armadas y Fuerzas y Cuerpos de Seguridad del Estado Español (34,5 44,2 años; $176,4 \pm 8,4 \mathrm{~cm} ; 74,6 \pm 8,7 \mathrm{~kg} ; 63,3 \pm 8,0 \mathrm{~kg}$ masa muscular; 7,6 23,2 kg masa grasa). Los sujetos pertenecían al Ejército de Tierra, Ejército del Aire, Armada, Guardia Civil y Cuerpo Nacional de Policía del Estado Español. Los participantes contaban con una permanencia profesional en sus respectivas unidades de entre 7 y 18 años, la mayoría de ellos tenían experiencia en misiones internacionales en los actuales conflictos de Líbano, Afganistán, Bosnia, Kosovo e Irak.

Los sujetos iban equipados con uniforme y botas reglamentarias, portaequipos de combate reglamentarios del Ejército de Tierra, arma corta de fuego simulada, machete reglamentario simulado, grilletes o bridas y una mochila de combate con $15 \mathrm{~kg}$ de peso, para simular el peso equivalente del equipo operativo para este tipo de actuaciones (antifragmentos o antibalas, casco, cargadores municionados, transmisiones, raciones de emergencia en su caso, paquete de cura individual, mapas y otros equipamientos básicos del combatiente).

\section{Simulación de combate}

El objetivo de la prueba es evaluar a los sujetos en una simulación de combate para valorar su respuesta ante una intervención con distintas incidencias, aplicando un uso proporcionado de la fuerza que puede oscilar entre el uso de la palabra, una acción de fuego, procedimientos operativos de engrilletado y la aplicación de técnicas de control, golpeo y derribo.

Los combatientes fueron divididos en 5 unidades de 4 combatientes cada una. Estos debían recorrer una pista en la que tenían que superar los siguientes obstáculos:

- Carrera de $100 \mathrm{~m}$ a ritmo aeróbico

- Obstáculo de triple barra en escalera (altura final 1,80 m)

- Paso de obstáculos sobre curso de agua (puente inclinado roto con una luz de $1 \mathrm{~m}$ y barras de equilibrio de $4 \mathrm{~m}$ )

- Espaldera de 2,2 m

- Foso con descenso por rampa y subida escalando $4 \mathrm{~m}$

- Paso de alambradas bajas $8 \mathrm{~m}$.

Después de sobrepasar estos obstáculos tenían que limpiar una trinchera en la que se encontraban con las siguientes incidencias:

- Sujeto que colabora con la unidad con un arma escondida

-- Sujeto armado con fusil que no entiende el idioma de la fuerza.

- Sujeto con estrés de combate que se encuentra llorando y tiene en sus inmediaciones un arma.

- Sujeto que intenta agredir con arma blanca a la fuerza a una distancia de $5 \mathrm{~m}$.

- Sujeto que dispara a la fuerza con arma corta de fogueo.

A continuación debían reducir a un centinela con arma blanca simulada que se encontraba vigilando la fachada de un edificio.

\section{Procedimiento}

Se realizaron las siguientes evaluaciones antes e inmediatamente después de la simulación de combate:

1. Percepción subjetiva de esfuerzo (RPE) con la escala 6 $20^{11}$.

2. Fuerza isométrica de la zona lumbar y piernas con el dinamómetro TKK. 5402. Takei Scientific Instruments CO LTD, con el que realizaban 2 repeticiones de contracción máxima, en la cual los sujetos debían realizar el movimiento de levantamiento de la barra de la galga isométrica, de ellas se utilizaba el máximo valor obtenido como parámetro de estudio.

3. Fuerza explosiva de piernas mediante sistema de células de infrarrojos Sistema Ergojump (Bosco System, Ergotest Technology) consistente en la realización de una batería de 6 saltos, 2 Squat Jump (SJ), 2 saltos con contramovimiento $(\mathrm{CMJ})$ y 2 saltos con contramovimiento y ayuda de brazos (ABK). Se utilizó una adaptación del protocolo de Clemente ${ }^{12}$

4. Lactato sanguíneo tomando una muestra de $32 \mu \mathrm{l}$ de sangre capilar de un dedo de los sujetos y analizado posteriormente con el sistema de lactato Dr. Lange. Miniphotometer plus LP 20. v 1.4.

5. Activación cortical y fatiga del Sistema Nervios Central (SNC) mediante el sistema Lafayette Instrument Flicker Fusion Control Unit (Model 12021). Con este sistema se 


\section{Respuesta orgánica en una simulación de combate}

realizaron 2 test diferentes, uno ascendente y otro descendente. En el primero, ascendente, el sujeto debía detectar el cambio de una luz discontinua a una luz continua. En el segundo test, descendente, el sujeto debía detectar el cambio de una luz continua a una luz discontinua. Cuando se detectaba el cambio en las luces el sujeto debía activar un pulsador ${ }^{13}$. Los sujetos realizaban dos veces cada uno de los test a las siguientes frecuencias:

- $1^{\circ}$ Test. 0 a $100 \mathrm{~Hz}$ : ascendente.

- $2^{\circ}$ Test. 100 a $0 \mathrm{~Hz}$ : descendente.

En cada uno de los test se cuantificó el tiempo que los sujetos tardaban en detectar los cambios en las luces desde el comienzo del test hasta el momento de activar el pulsador, para poder determinar el Umbral de Frecuencia Crítica de Fusión (UFCF) entendido éste como la media de los valores obtenidos en el test ascendente y la media de los valores obtenidos en el test descendente.

\section{Análisis Estadístico}

El análisis estadístico se realizó con el programa estadístico SPSS 17.0. Primero se determino la normalidad de la muestra con la prueba de Kolmogorov-Smirnof. A continuación se realizó un análisis de la varianza con un post hoc de Bonferroni. Para todas las comparaciones se aceptó el índice de significación de $\mathrm{p}<0,05$.

\section{RESULTADOS}

Al estudiar los datos obtenidos observamos como el valor del UFCF disminuyó de $37,11 \pm 3,40 \mathrm{~Hz}$ a $36,77 \pm 4,54 \mathrm{~Hz}$ $(-0,91 \%)$. Los resultados de fuerza isométrica aumentaron ligeramente desde $142,47 \pm 20,54 \mathrm{~N}$ a $147,66 \pm 26,66 \mathrm{~N}$ después de la simulación $(3,64 \%)$. Los valores de percepción subjetiva de esfuerzo fueron de 14,5 $\pm 2,0$. La concentración sanguínea de lactato aumento significativamente $(\mathrm{p}<0,05)$ desde $2,57 \pm 0,62 \mathrm{mmol} / 1$ hasta 4,92 $\pm 2,18 \mathrm{mmol} / \mathrm{l}$ al finalizar la simulación de combate $(91,4 \%)$. Los resultados de fuerza explosiva de piernas muestran una disminución significativa $(\mathrm{p}<0,05)$ de todos los valores como se muestra en la tabla 1.

Tabla 1. Resultados de los parámetros analizados en la simulación de combate.

\begin{tabular}{|ccccc|}
\hline & Unidad & PRE & POST & \% Cambio \\
\hline UFCF & $\mathrm{Hz}$ & $37,11 \pm 3,40$ & $36,77 \pm 4,54$ & $-0,91$ \\
F. Isométrica & $\mathrm{N}$ & $142,47 \pm 20,54$ & $147,66 \pm 26,66$ & 3,64 \\
RPE & - & - & $14,5 \pm 2,0$ & - \\
Lactato & $\mathrm{mmol} / 1$ & $2,57 \pm 0,62$ & $4,92 \pm 2,18$ & $91,4^{*}$ \\
SJ & $\mathrm{m}$ & $0,27 \pm 0,06$ & $0,25 \pm 0,10$ & $-7,09^{*}$ \\
CMJ & $\mathrm{m}$ & $0,30 \pm 0,06$ & $0,29 \pm 0,10$ & $-2,64^{*}$ \\
ABK & $\mathrm{m}$ & $0,35 \pm 0,06$ & $0,33 \pm 0,08$ & $-5,47^{*}$ \\
\hline
\end{tabular}

UFCF - Umbral de Frecuencia Crítica de Fusión. *p<0,05.
Los combatientes realizaron una media de 378,3 $\pm 92,6$ metros recorridos, con una velocidad media de desplazamiento de $1,9 \pm 0,6 \mathrm{~m} / \mathrm{s}$ para la realización de la simulación de combate.

\section{DISCUSIÓN}

El análisis de los resultados obtenidos muestra como esta simulación de combate provoca una respuesta cortical alta provocando una disminución de los valores de UFCF, lo que pone de manifiesto que este tipo de situaciones de combate puede provocar ligeros síntomas de fatiga del sistema nervioso central ${ }^{14}$. Esta respuesta es similar a la evaluada en sujetos después de realizar pruebas de velocidad ${ }^{15}$ o después de realizar una prueba de esfuerzo hasta la extenuación ${ }^{16}$. Los resultados obtenidos muestran el incremento en la activación cortical que representa una situación de combate, en donde los combatientes tienen que superar una serie de obstáculos y hacer frente a una situación en la que existen un gran número de incertidumbres (como la limpieza de la trinchera o la neutralización del centinela). Estas situaciones pueden ser interpretadas por el cerebro como posibles elementos de los cuales puede surgir una respuesta hostil y por lo tanto, se puede poner en peligro la integridad del sujeto, suponiendo para él una amenaza. Esta situación puede provocar en el sujeto un estado de ansiedad ${ }^{17}$ en el que el cerebro se ve sobreexcitado ante todos estos estímulos y puede llegar a convertirse en una situación muy estresante para él. Este aumento en el grado de atención y de activación ante esta situación estresante hace que el organismo este en un continuo estado de alerta para poder responder rápidamente ante cualquier estimulo que se le presente. Este alto grado de activación y tensión muscular se ve reflejada en los valores de lactato sanguíneo, los cuales aumentaron significativamente hasta llegar a valores superiores a los del umbral anaeróbico $(4,92 \pm 2,18 \mathrm{mmol} / 1)^{18}$. Este gran aumento de los valores de lactato sanguíneo también fue originado por el componente físico que conlleva el paso de los diferentes obstáculos cargando con el equipo que realizaron los combatientes, aunque la velocidad media para la realización del ejercicio fue muy baja $(1,9 \pm 0,6 \mathrm{~m} / \mathrm{s})$.

A pesar del elevado nivel de lactato sanguíneo y los síntomas de fatiga del SNC mostrada por los sujetos de esta investigación el valor de RPE fue de 14,5 $\pm 2,0$, valor que no se corresponde con ninguno de los dos parámetros anteriormente comentados (lactato y fatiga del SNC). A raíz de estos resultados podemos pensar que los sujetos no son conscientes realmente de la carga psicológica ni fisiológica que provoca una situación de combate, mostrando una percepción subjetiva de esfuerzo más acorde con la carga externa realizada por ellos durante la acción (movimientos a baja velocidad).

$\mathrm{Si}$ analizamos los valores obtenidos en la fuerza isométrica podemos ver como aumentaron después de la simulación de combate. Este aumento de los valores de fuerza de los sujetos puede ser debido al aumento de la activación cortical, que a pesar de ser tan elevada que mostraba síntomas de fatiga del SNC ha podido ser el responsable de este aumento de los valores de fuerza. Este hecho puede tener su explicación en los mecanismos de defensa del cuerpo humano como es la respuesta de luchahuida en la cual el sistema nervioso simpático es activado y pre- 
para al cuerpo para cualquier situación de peligro ${ }^{19}$. En el caso de esta investigación, el organismo de los combatientes que participaron en ella interpreta que la situación de combate es origen de estímulos que pueden considerarse peligrosos o que pueden poner en peligro la integridad física del organismo por lo tanto se activa esta respuesta de lucha-huida en la cual el sistema simpático produce una mayor activación muscular. Esta activación muscular, a su vez produce un aumento de la fuerza muscular de los sujetos, que se ha mostrado en el aumento de los valores de fuerza isométrica. A pesar de ello, los resultados de fuerza explosiva de piernas disminuyeron debido a que el paso de los obstáculos suponía un trabajo principalmente de la musculatura de los miembros inferiores, a lo que hay que sumar el peso del equipo, por lo que este trabajo muscular total ha podido producir fatiga muscular en los miembros inferiores, situación que ha provocado esa disminución en los valores de fuerza explosiva de piernas.

\section{Aplicación práctica}

Los resultados obtenidos han puesto de manifiesto la respuesta del organismo de los combatientes en una situación simulada de combate.

Con estos resultados se pueden llevar a cabo planes de entrenamientos específicos aplicados a las intervenciones operativas de carácter militar en situaciones de estrés. Debido a los altos niveles de lactato sanguíneo mostrados en esta prueba, la realización de trabajos de tolerancia láctica o de aclaración de lactato mejoraría el rendimiento de los sujetos. Posibilitando una mejor actuación en este tipo de situaciones especificas de combate.

Los datos de fatiga del sistema nervioso central obtenidos ponen de manifiesto la necesidad de perfeccionar los sistemas de entrenamiento de gestión del estrés, para permitir disminuir los valores de fatiga del $\mathrm{SNC}$, con entrenamientos específicos para una mejor actuación en las actuales situaciones de combate. Además, nos inducen a pensar en la importancia del entrenamiento psicológico y la capacidad para poder gestionar el estrés, abriéndose la posibilidad de cuantificar la carga de la fatiga del sistema nervioso central y contrastarla con el rendimiento del combatiente. Estos parámetros podrían estar relacionados con el efecto túnel y el tiempo de decisión y reacción en una situación de combate cuerpo a cuerpo, factores que serían interesantes de analizar en futuras investigaciones.

\section{CONCLUSIÓN}

Después de analizar los datos se puede concluir que una simulación de combate se realiza con una concentración de lactato sanguíneo superior al umbral anaeróbico. Además, se pone de manifiesto el alto grado de estrés de este tipo de combate, ya que los sujetos acaban con síntomas de fatiga del SNC. Por último, a pesar de los síntomas de fatiga del SNC los combatientes son capaces de desarrollar valores de fuerza superiores a los mostrados en condiciones basales en la musculatura que no han estado implicados principalmente en el paso de obstáculos.

\section{BIBLIOGRAFÍA}

1. Murray S. El mando en combate, la toma de decisiones y el panopticon del campo de batalla. Military Review 2003; Enero-Febrero: 12-19.

2. Metz S. Asimetría estratégica. Military Review 2002; Mayo-Junio: 64-73.

3. Braganca E. La evolución de fuegos conjuntos. Military Review 2004; JulioAgosto: 51-55.

4. Calvo JL. ¿Qué ha fallado en Iraq?. Revista Ejército 2005; 766.16-21

5. Lester M, Knapikt J, Catrambone D, Antczak A, Sharp M, Burrel L, Darakhy S. Effect of a 13-month deployment to Iraq on physical fitness and body composition. Military Medicine 2010; 176(6): 417-423.

6. Ricciardi R, Deuster P, Talbot L. Effect of gender and body adiposity on physiological responses to physical work while wearing body armor. Military Medicine 2007; 172(7): 743-748.

7. Rintamäki H, Oksa J, Rissanen S, Mäkinen T, Kyröläinen H, Keskinen O, Kauranen K, Peitso A. Physical Activity during a 12 Days Military Field Training in Winter and the Effects on Muscular and Cardiorespiratory Fitness. In Strategies to Maintain Combat Readiness during Extended Deployments - A Human Systems Approach 2005 (pp. 18-1 - 18-6). Meeting Proceedings RTO-MP-HFM-124, Paper 18. Neuilly-sur-Seine, France: RTO.

8. Simpson R, Gray S, Geraint D. Physiological variables and performance markers of serving soldiers from two "elite" units of the British Army. Journal of Sports Science 2006; 24(6): 597-604.

9. Harman E, Gutekunst D, Frykman P, Sharp M, Nindl B, Alemany J, Mello R. Prediction of simulated battlefield physical performance from field-expedient test. Military Medicine 2008; 173(1):36-41.

10. Jiménez F. Estudio descriptivo y valoración del gasto energético en actividades militares de combate simulado. 2002. Tesis doctoral. Universidad de Cordoba. Cordoba.

11. Borg G. Perceived exertion as an indicator of somatic stress. Scandinavian Journal of Rehabilitation Medicine1970; 2(2), 92-98.

12. Clemente V. Efectos de la distribución y secuencia en la organización de distintas tareas de entrenamiento para la mejora de la resistencia aeróbica. 2011. Tesis doctoral. Universidad Castilla La Mancha. Toledo.

13. Davranche K, Pichon A. Critical Flicker Frequency Threshold Incerment After an Exhausting Exercise. Journal of Sport \& Exercise Psychology 2005; 27: 515-520.

14. Li Z, Jiao K, Chen M, Wang C. Reducing the effects of driving fatigue with magnitopuncture stimulation. Accident Analysis and Prevention 2004; 36 : 501-505

15. Clemente V, Muñoz V, Melús M.. Fatiga del sistema nervioso después de realizar un test de capacidad de sprints repetidos (RSA) en jugadores de futbol profesionales. Arch Med Deporte. 2011; 143, 103-112.

16. Godefroy D, Rousseu C, Vercruyssen F, Cremieux J, Brisswalter J. Influence of physical exercise on perceptual response in aerobically trained subjects Percept Mot Skills. 2002; 94: 68-70.

17. Martens R, Vealey R, Burton D. Competitive anxiety in sport. Champaign: Human kinetics, 1990. 342-345.

18. Sjödin B, Jacobs I. Onset of blood lactate accumulation and marathon running performance. International Journal of Sports Medicine 1981; 2:23-26.

19. Sandín B. El estrés: un análisis basado en el papel de los factores sociales. Internacional Journal of Clinical and Health Psychology 2003; 3(1):141-157 